\title{
Percutaneous placement of intra-aortic balloon pump in the left axillary position improving quality of care in severe heart failure
}

\section{Colocación percutánea de balón de contrapulsación intraaórtico para mejorar la calidad de cuidado en falla cardiaca grave}

\author{
Jaime A. Guajardo-Lozano ${ }^{1}$, José G. Paredes-Vázquez", Héctor López-de la Garza1, \\ Carlos Jerjes-Sánchez ${ }^{1,2}$, Juan A. Quintanilla1, and Guillermo Torre-Amione1,2*
}

${ }^{1}$ Tecnológico de Monterrey, Escuela de Medicina y Ciencias de la Salud, Monterrey, N.L., México; ${ }^{2}$ Instituto de Cardiología y Medicina Vascular, TecSalud, San Pedro Garza García, N.L., México

\section{Introduction}

The most common approach to intra-aortic balloon pumps (IAPB) percutaneous placement is the transfemoral artery inducing physical deconditioning in prolonged support conditions ${ }^{1}$. We previously described the percutaneous technique of installation of an IABP through the left axillary or subclavian artery as a feasible and relatively well-tolerated approach to bridge patients with end-stage heart failure (HF) to heart transplantation'. We present - to the best of our knowledge - the first case of axillary IABP percutaneous placement in Mexico. This case reproduces the feasibility and tolerability of the axillary approach in a patient with acute severe HF as a bridge to the left ventricular assist device (LVAD) therapy.

\section{Case report}

A 72-year-old male was admitted to ER with a new episode of progressive dyspnea. He had a history of ischemic cardiomyopathy, surgical revascularization, HF with reduced ejection fraction (HFrEF) (35\% ejection fraction $[\mathrm{EF}$ ) with multiple exacerbations, paroxysmal atrial fibrillation (AF), diabetes, and chronic kidney disease (CKD). The previous treatment including bumetanide, spironolactone, aspirin, ivabradine, amiodarone, apixaban, and rosuvastatin. Physical examination with HR 102 bpm, respiratory rate $24 \mathrm{bpm}$, blood pressure $98 / 66 \mathrm{mmHg}$, oxygen saturation of $89 \%$, and venous distension. The chest auscultation revealed S3, bilateral rales, right pleural effusion, and anasarca. The electrocardiogram and chest X-ray showed ischemic findings and congestive lungs with the right pleural effusion, respectively. Laboratories demonstrate hemoglobin $10.7 \mathrm{~g} / \mathrm{dL}$, B-type natriuretic peptide $1672 \mathrm{pg} / \mathrm{mL}$, and creatinine $4.4 \mathrm{mg} / \mathrm{dL}$. Transthoracic echocardiography showed severe global ventricular hypokinesis, moderate mitral regurgitation, left atrium enlargement with a volume of $34 \mathrm{ml} / \mathrm{m}^{2}$, diastolic dysfunction, pulmonary artery systolic pressure $59 \mathrm{mmHg}$, and $21 \% \mathrm{EF}$. We established the diagnosis of acute HF and started $40 \mathrm{mg}$ of intravenous furosemide and milrinone $0.25 \mathrm{mcg} / \mathrm{kg} / \mathrm{min}$. We performed thoracentesis of $1800 \mathrm{cc}$, and by medical treatment failure, we increased the milrinone dose to $0.5 \mathrm{mcg} / \mathrm{kg} / \mathrm{min}$ and start $2 \mathrm{mg}$ of bumetanide every $6 \mathrm{~h}$ without success. We started hemodialysis and amiodarone for paroxysmal $\mathrm{AF}$ and
Correspondence:

*Guillermo Torre-Amione

E-mail: gtorre@tecsalud.mx

1405-9940/๑ 2020 Instituto Nacional de Cardiología Ignacio Chávez. Published by Permanyer. This is an open access article under the CC BY-NC-ND license (http://creativecommons.org/licenses/by-nc-nd/4.0/).
Date of reception: 27-11-2019

Chávez. Published by Permanyer. This is an open access article under the CC BY-NC-ND
Available online: $23-12-2020$ Arch Cardiol Mex. 2020;90(4):539-541
Date of acceptance: 02-04-2020

.




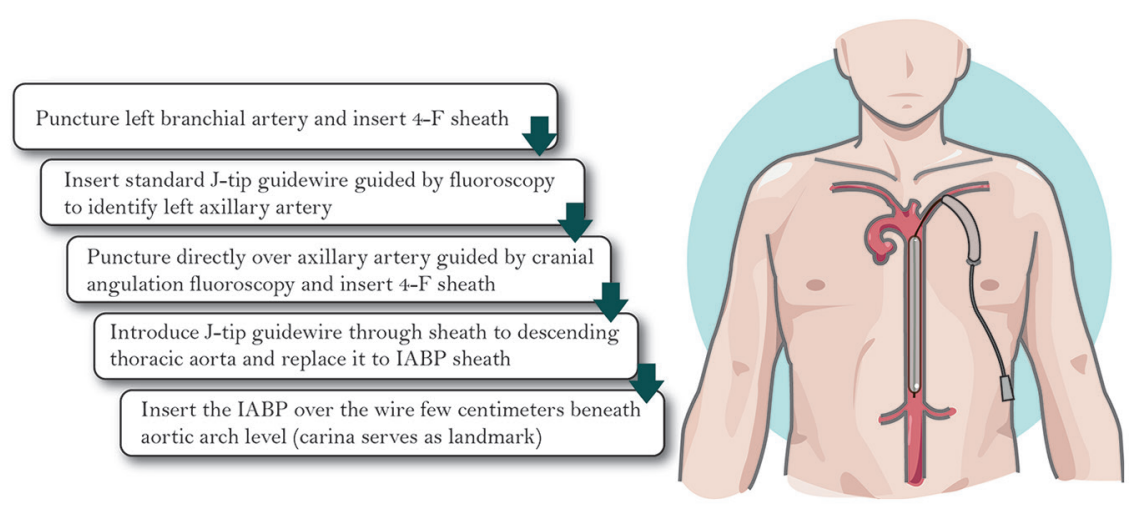

Figure 1. Description of the main steps for the percutaneous placement of an intra-aortic balloon pump in the left axillary/subclavian position.

an episode of self-limiting ventricular monomorphic tachycardia, reverting to sinus rhythm. In the following hours, we started norepinephrine by worsening clinical instability and severe hypotension. Because of persistent amine dependency and poor inotropic response, we perform a right cardiac catheterization as the protocol for LVAD showing pressures of the right atrium $(20 \mathrm{mmHg})$, right ventricle $(70 / 10 \mathrm{mmHg})$, pulmonary artery (70/30 $\mathrm{mmHg})$, pulmonary capillary wedge pressure (PCWP) of $29 \mathrm{mmHg}$, a right atrial pressure/PCWP 0.9 and a pulmonary artery pulsatility index of 4 , and a cardiac index of $1.7 \mathrm{~L} / \mathrm{min}$, with persistent clinical instability and treatment failure. The patient was classified as a D-stage shock, according to the Society for Cardiovascular Angiography and Interventions cardiogenic shock consensus ${ }^{2}$. During follow-up, the patient continued with inotropic and chronotropic agents with an impending need for the left ventricular support and hemodialysis with total ultrafiltration of $6 \mathrm{~L}$.

In addition, a femoral Doppler ultrasound showed critical stenosis $90 \%$ in the right and left proximal femoral common artery, distal occlusion of the common femoral artery (calcified plaques). Considering these findings, we decided the percutaneous axillary approach. A left brachial and axillary Doppler ultrasound showed adequate blood flow without critical stenosis. The patient was considered as bridge therapy for LVAD or cardiac transplantation, with an expected extended in-hospital stay and under medical treatment waiting to transfer to a specialized HF center.

\section{Technique description}

We previously described the most extensive series and the technique of percutaneous placement of IABP in the axillary/subclavian ${ }^{1}$ (Fig. 1).

\section{In-hospital outcome}

After the placement of the axillary IABP, heart and renal function improved. Physical therapy interventions were focused on preventing weakness, early ambulation, and stand-up position exercises (Fig. 2). Despite the patient, PCWP $(24 \mathrm{mmHg})$, and cardiac index (2.7 L/min) improvement, he was rejected for an LVAD or a heart transplant.

The clinical outcome after IABP removal was that of a terminal HF patient rejected to advanced treatment options. The patient eventually passed away despite optimal medical treatment.

\section{Discussion}

This case showed that the axillary approach IABP in patients with advanced HFrEF is safe and improves the quality of life, and reproduces previous evidence in under urgent heart transplant ${ }^{3}$. The left axillary/subclavian $I A B P^{1}$ is an option that reaches the benefits of the conventional IABP femoral access reducing complications secondary to immobility and avoids a surgical cut-down and general anesthesia mandatory in intrathoracic surgical approaches ${ }^{3,4}$. Previously, we described the safety and efficacy of axillar/subclavian IABP in 50 patients with HFrEF under heart or heart-multiorgan transplantation. Cumulative survival on IABP support was $92 \%$; $8 \%$ had thromboembolic or bleeding events. Four patients died during the support of secondary multiorgan failure. Our case was complex by multiple comorbidities, severe peripheral arterial disease, hemodynamic instability, vasopressor and inotrope dependence, and highrisk complications with LVAD insertion. We performed the axillary approach successfully ${ }^{5}$, achieving clinical 


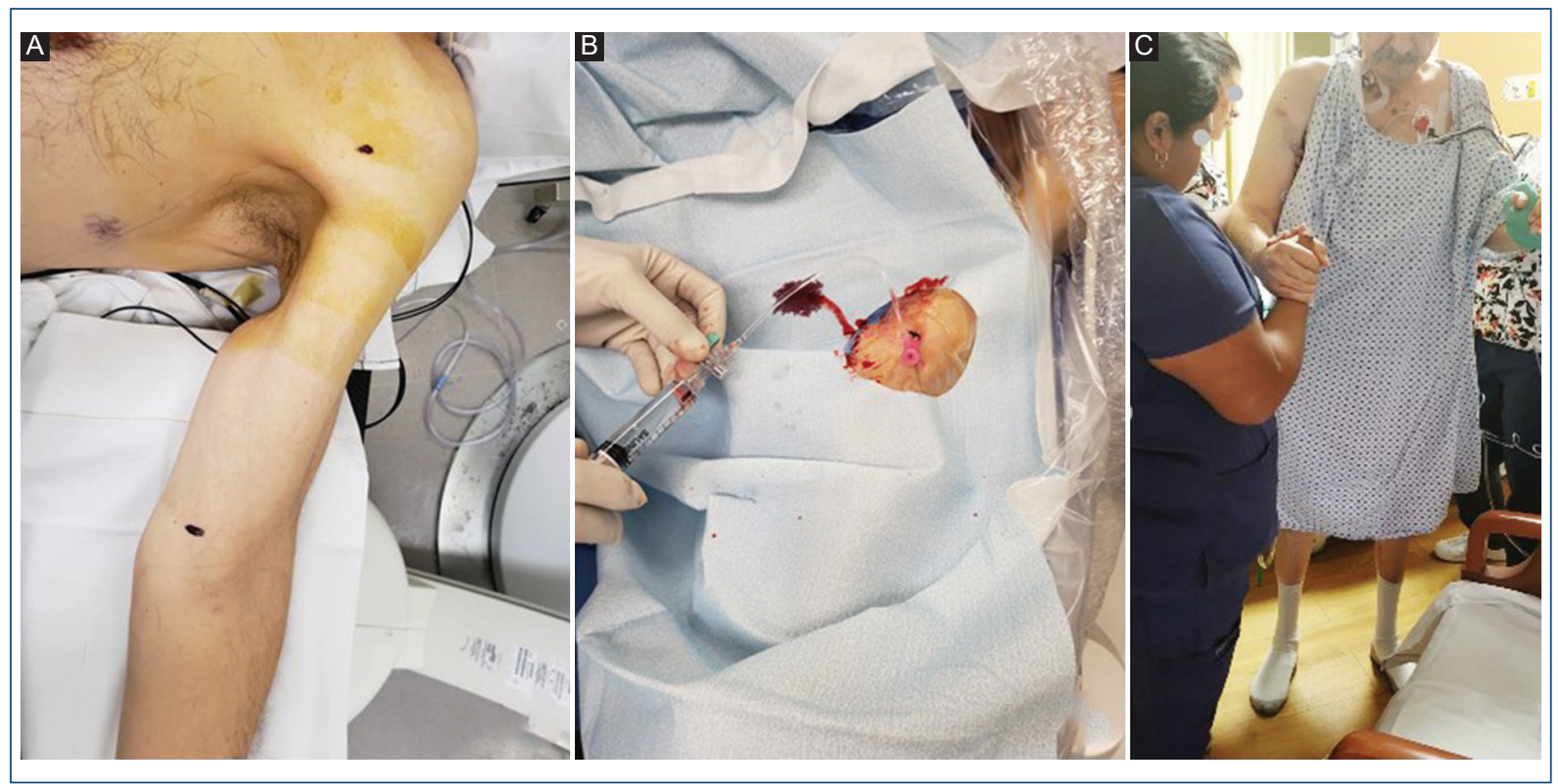

Figure 2. The axillary route improves the patient's quality of life.

stability, renal function, and quality of life improvement without bleeding or procedure-related complications. We recommend the left axillary artery route instead of the right access to avoid the cerebral artery vessels ${ }^{6}$. The axillar percutaneous approach could be an option in patients with extensive and severe PAD or those with expected long time to destination therapy.

\section{Funding}

The present investigation is not funded by any specific scholarship from public, commercial, or profit agencies.

\section{Conflicts of interest}

The authors declare no conflicts of interest.

\section{Ethical disclosures}

Protection of human and animal subjects. The authors declare that no experiments were performed on humans or animals for this study.

Confidentiality of data. The authors declare that they have followed the protocols of their work center on the publication of patient data.
Right to privacy and informed consent. The authors have obtained the written informed consent of the patients or subjects mentioned in the article. The corresponding author is in possession of this document.

\section{References}

1. Estep JD, Cordero-Reyes AM, Bhimaraj A, Trachtenberg B, Khalil N Loebe $M$, et al. Percutaneous placement of an intra-aortic balloon pump in the left axillary/subclavian position provides safe, ambulatory longterm support as bridge to heart transplantation. JACC Heart Fail. 2013;1:382-8

2. Baran DA, Grines CL, Bailey S, Burkhoff D, Hall SA, Henry TD, et al. SCAI clinical expert consensus statement on the classification of cardiogenic shock: this document was endorsed by the American College of Cardiology (ACC), the American Heart Association (AHA), the Society of Critical Care Medicine (SCCM), and the Society of Thoracic Surgeons (STS) in April 2019. Catheter Cardiovasc Interv. 2019;94:29-37.

3. Barge-Caballero E, González-Vílchez F, Delgado JF, Mirabet-Pérez S, González-Costello J, Pérez-Villa F, et al. Use of intra-aortic balloon pump as a bridge to heart transplant in Spain: results from the ASIS-TC study. Rev Esp Cardiol (Engl Ed). 2019;72:835-43.

4. Cheng JM, den Uil CA, Hoeks SE, van der Ent M, Jewbali LS, van Domburg RT, et al. Percutaneous left ventricular assist devices vs. intra-aortic balloon pump counterpulsation for treatment of cardiogenic shock: a meta-analysis of controlled trials. Eur Heart J. 2009;30:2102-8.

5. Umakanthan R, Hoff SJ, Solenkova N, Wigger MA, Keebler ME, Lenneman A, et al. Benefits of ambulatory axillary intra-aortic balloon pump for circulatory support as bridge to heart transplant. J Thorac Cardiovasc Surg. 2012;143:1193-7.

6. Russo MJ, Jeevanandam V, Stepney J, Merlo A, Johnson EM, Malyala $\mathrm{R}$, et al. Intra-aortic balloon pump inserted through the subclavian artery: a minimally invasive approach to mechanical support in the ambulatory end-stage heart failure patient. J Thorac Cardiovasc Surg. 2012;144:951-5. 